Homology, Homotopy and Applications, vol.13(1), 2011, pp.259-272

\title{
OPERATIONS ON THE HOPF-HOCHSCHILD COMPLEX FOR MODULE-ALGEBRAS
}

\author{
DONALD YAU
}

(communicated by Claude Cibils)

\begin{abstract}
It is proved that Kaygun's Hopf-Hochschild cochain complex for a module-algebra is a brace algebra with multiplication. As a result, an analogue of Deligne's Conjecture holds for modulealgebras, and the Hopf-Hochschild cohomology of a modulealgebra has a Gerstenhaber algebra structure.
\end{abstract}

\section{Introduction}

Let $H$ be a bialgebra, and let $A$ be an associative algebra. The algebra $A$ is said to be an $H$-module-algebra if there is an $H$-module structure on $A$ such that the multiplication on $A$ becomes an $H$-module morphism. For example, if $S$ denotes the Landweber-Novikov algebra $[\mathbf{1 5}, \mathbf{2 2}]$, then the complex cobordism $M U^{*}(X)$ of a topological space $X$ is an $S$-module-algebra. Likewise, the singular mod $p$ cohomology $H^{*}\left(X ; \mathbf{F}_{p}\right)$ of a topological space $X$ is an $\mathcal{A}_{p}$-module-algebra, where $\mathcal{A}_{p}$ denotes the Steenrod algebra associated to the prime $p[\mathbf{7}, \mathbf{2 0}]$. Other similar examples from algebraic topology can be found in [4]. Important examples of module-algebras from Lie and Hopf algebras theory can be found in, e.g., [12, V.6].

In [14] Kaygun defined a Hochschild-like cochain complex $\mathrm{CH}_{\mathrm{Hopf}}^{*}(A, A)$ associated to an $H$-module-algebra $A$, called the Hopf-Hochschild cochain complex, that takes into account the $H$-linearity. In particular, if $H$ is the ground field, then Kaygun's Hopf-Hochschild cochain complex reduces to the usual Hochschild cochain complex $C^{*}(A, A)$ of $A[\mathbf{1 1}]$. Kaygun [14] showed that the Hopf-Hochschild cohomology of $A$ shares many properties with the usual Hochschild cohomology. For example, it can be described in terms of derived functors, and it satisfies Morita invariance.

The usual Hochschild cochain complex $C^{*}(A, A)$ has a very rich structure. Namely, it is a brace algebra with multiplication [10]. Combined with a result of McClure and Smith [19] concerning the singular chain operad associated to the little squares operad $\mathcal{C}_{2}$, the brace algebra with multiplication structure on $C^{*}(A, A)$ leads to a positive solution of Deligne's Conjecture [6]. Also, passing to cohomology, the brace algebra with multiplication structure implies that the Hochschild cohomology modules $H H^{*}(A, A)$ form a Gerstenhaber algebra, which is a graded version of a Poisson algebra. This fact was first observed by Gerstenhaber $[\mathbf{8}]$.

Received March 8, 2011, revised April 10, 2011; published on May 22, 2011.

2000 Mathematics Subject Classification: 16E40, 16W30.

Key words and phrases: module-algebra, Hopf-Hochschild cohomology, Gerstenhaber algebra, brace algebra, Deligne's conjecture.

Article available at http://intlpress.com/HHA/v13/n1/a9 and doi:10.4310/HHA.2011.v13.n1.a9

Copyright (C) 2011, International Press. Permission to copy for private use granted. 
The purpose of this note is to observe that Kaygun's Hopf-Hochschild cochain complex $\mathrm{CH}_{\mathrm{Hopf}}^{*}(A, A)$ of a module-algebra $A$ also admits the structure of a brace algebra with multiplication. In fact, the Hopf-Hochschild complex is a sub-complex of the usual Hochschild complex, and the former inherits the latter's structure. As in the classical case, this leads to a version of Deligne's Conjecture for module-algebras. Also, the Hopf-Hochschild cohomology modules $H H_{\text {Hopf }}^{*}(A, A)$ form a Gerstenhaber algebra. When the bialgebra $H$ is the ground field, these structures reduce to the ones in Hochschild cohomology.

A couple of remarks are in order. First, there is another cochain complex $C_{M A}^{*}(A)$ that can be associated to an $H$-module-algebra $A[\mathbf{2 4}]$. The cochain complex $C_{M A}^{*}(A)$ is different from the Hopf-Hochschild complex $\mathrm{CH}_{\text {Hopf }}^{*}(A, A)$. The former controls the deformations of $A$, in the sense of Gerstenhaber [9], with respect to both the $H$-module structure and the algebra structure on $A$. It is not known if there is any relationship between the two complexes.

Second, the results and arguments here can be adapted to module-coalgebras, comodule-algebras, and comodule-coalgebras. To do that, one replaces the crossed product algebra $X$ (section 2.3) associated to an $H$-module-algebra $A$ by a suitable crossed product (co)algebra $[\mathbf{1}, \mathbf{2}, \mathbf{3}]$ and replaces Kaygun's Hopf-Hochschild cochain complex by a suitable variant.

\subsection{Organization}

The rest of this paper is organized as follows.

In the following section, we recall the construction of the Hopf-Hochschild cochain complex $\mathrm{CH}_{\mathrm{Hopf}}^{*}(A, A)$ from Kaygun [14].

In Section 3, it is observed that $\mathrm{CH}_{\mathrm{Hopf}}^{*}(A, A)$ has the structure of an operad with multiplication (Theorem 3.1). This leads in Section 4 to the desired brace algebra with multiplication structure on $\mathrm{CH}_{\mathrm{Hopf}}^{*}(A, A)$ (Corollary 4.1). Explicit formulas of these brace operations are given (11).

In Section 5, it is observed that the brace algebra with multiplication structure on $\mathrm{CH}_{\text {Hopf }}^{*}(A, A)$ leads to a homotopy $G$-algebra structure (Corollary 5.1). The differential from this homotopy $G$-algebra and the Hopf-Hochschild differential are then identified, up to a sign (Theorem 5.2).

Passing to cohomology, this leads in Section 6 to a Gerstenhaber algebra structure on the Hopf-Hochschild cohomology modules $H H_{\text {Hopf }}^{*}(A, A)$ (Corollary 6.1). The graded associative product and the graded Lie bracket on $H H_{\text {Hopf }}^{*}(A, A)$ are explicitly described (16).

In the final section, combining our results with a result of McClure and Smith [19], a version of Deligne's Conjecture for module-algebras is obtained (Corollary 7.1). This section can be read immediately after Section 4 and is independent of Sections 5 and 6 .

\section{Acknowledgements}

The author thanks the anonymous referee for his/her helpful suggestions. 


\section{Hopf-Hochschild cohomology}

In this section, we fix some notations and recall from [14, Section 3] the HopfHochschild cochain complex associated to a module-algebra.

\subsection{Notations}

Fix a ground field $K$ once and for all. Tensor product and vector space are all meant over $K$.

Let $H=\left(H, \mu_{H}, \Delta_{H}\right)$ denote a $K$-bialgebra with associative multiplication $\mu_{H}$ and coassociative comultiplication $\Delta_{H}$. It is assumed to be unital and counital, with its unit and counit denoted by $1_{H}$ and $\varepsilon: H \rightarrow K$, respectively.

Let $A=\left(A, \mu_{A}\right)$ denote an associative, unital $K$-algebra with unit $1_{A}$ (or simply $1)$.

In a coalgebra $(C, \Delta)$, we use Sweedler's notation $[\mathbf{2 3}]$ for comultiplication:

$$
\Delta(x)=\sum x_{(1)} \otimes x_{(2)}, \Delta^{2}(x)=\sum x_{(1)} \otimes x_{(2)} \otimes x_{(3)}, \text { etc. }
$$

These notations will be used throughout the rest of this paper.

\subsection{Module-algebra}

Recall that the algebra $A$ is said to be an $H$-module-algebra $[\mathbf{5}, \mathbf{1 2}, \mathbf{2 1}, \mathbf{2 3}]$ if and only if there exists a left $H$-module structure on $A$ such that $\mu_{A}$ and the unit map $1_{A}: K \rightarrow A$ are both $H$-module morphisms, i.e.,

$$
b\left(a_{1} a_{2}\right)=\sum\left(b_{(1)} a_{1}\right)\left(b_{(2)} a_{2}\right)
$$

for $b \in H$ and $a_{1}, a_{2} \in A$, and

$$
b\left(1_{A}\right)=\varepsilon(b) 1_{A}
$$

for $b \in H$.

We will assume that $A$ is an $H$-module-algebra for the rest of this paper.

\subsection{Crossed product algebra}

Define the vector space

$$
X \stackrel{\text { def }}{=} A \otimes A \otimes H .
$$

Define a multiplication on $X[\mathbf{1 4}$, Definition 3.1$]$ by setting

$$
\left(a_{1} \otimes a_{1}^{\prime} \otimes b^{1}\right)\left(a_{2} \otimes a_{2}^{\prime} \otimes b^{2}\right)=\sum a_{1}\left(b_{(1)}^{1} a_{2}\right) \otimes\left(b_{(3)}^{1} a_{2}^{\prime}\right) a_{1}^{\prime} \otimes b_{(2)}^{1} b^{2}
$$

for $a_{1} \otimes a_{1}^{\prime} \otimes b^{1}$ and $a_{2} \otimes a_{2}^{\prime} \otimes b^{2}$ in $X$. It is shown in [14, Lemma 3.2] that $X$ is an associative, unital $K$-algebra, called the crossed product algebra.

Note that if $H=K(=$ the trivial group bialgebra $K[\{e\}])$, then $X$ is just the enveloping algebra $A \otimes A^{\mathrm{op}}$, where $A^{\mathrm{op}}$ is the opposite algebra of $A$.

The algebra $A$ is a left $X$-module via the action

$$
\left(a \otimes a^{\prime} \otimes b\right) a_{0}=a\left(b a_{0}\right) a^{\prime}
$$

for $a \otimes a^{\prime} \otimes b \in X$ and $a_{0} \in A$. Likewise, the vector space $A^{\otimes(n+2)}$ is a left $X$-module 
via the action

$$
\left(a \otimes a^{\prime} \otimes b\right)\left(a_{0} \otimes \cdots \otimes a_{n+1}\right)=\sum a b_{(1)} a_{0} \otimes b_{(2)} a_{1} \otimes \cdots \otimes b_{(n+1)} a_{n} \otimes b_{(n+2)} a_{n+1} a^{\prime}
$$

for $a_{0} \otimes \cdots \otimes a_{n+1} \in A^{\otimes(n+2)}$.

\subsection{Bar complex}

Consider the chain complex $\mathrm{CB}_{*}(A)$ of vector spaces with

$$
\mathrm{CB}_{n}(A)=A^{\otimes(n+2)},
$$

and with differential

$$
d_{n}^{\mathrm{CB}}=\sum_{j=0}^{n}(-1)^{j} \partial_{j}: \mathrm{CB}_{n}(A) \rightarrow \mathrm{CB}_{n-1}(A),
$$

where

$$
\partial_{j}\left(a_{0} \otimes \cdots \otimes a_{n+1}\right)=a_{0} \otimes \cdots \otimes\left(a_{j} a_{j+1}\right) \otimes \cdots \otimes a_{n+1} .
$$

It is mentioned above that each vector space $\mathrm{CB}_{n}(A)=A^{\otimes(n+2)}$ is a left $X$-module. Using the module-algebra condition (1), it is straightforward to see that each $\partial_{j}$ is $X$-linear. Therefore, $\mathrm{CB}_{*}(A)$ can be regarded as a chain complex of left $X$-modules.

Note that in the case $H=K$, the chain complex $\mathrm{CB}_{*}(A)$ of $A \otimes A^{\mathrm{op}}$-modules is the usual bar complex of $A$.

\subsection{Hopf-Hochschild cochain complex}

The Hopf-Hochschild cochain complex of $A$ with coefficients in $A$ is the cochain complex of vector spaces:

$$
\left(\mathrm{CH}_{\mathrm{Hopf}}^{*}(A, A), d_{\mathrm{CH}}\right) \stackrel{\text { def }}{=} \operatorname{Hom}_{X}\left(\left(\mathrm{CB}_{*}(A), d^{\mathrm{CB}}\right), A\right) .
$$

Its $n$th cohomology module is denoted by $H H_{\mathrm{Hopf}}^{n}(A, A)$ and is called the $n$th HopfHochschild cohomology of $A$ with coefficients in $A$.

When $H=K$, the cochain complex $\left(\mathrm{CH}_{\mathrm{Hopf}}^{*}(A, A), d_{\mathrm{CH}}\right)$ is the usual Hochschild cochain complex of $A$ with coefficients in itself [11] and $H H_{\mathrm{Hopf}}^{n}(A, A)$ is the usual Hochschild cohomology module.

In what follows, we will use the notation $\mathrm{CH}_{\mathrm{Hopf}}^{*}(A, A)$ to denote (i) the HopfHochschild cochain complex $\left(\mathrm{CH}_{\mathrm{Hopf}}^{*}(A, A), d_{\mathrm{CH}}\right)$, (ii) the sequence $\left\{\mathrm{CH}_{\mathrm{Hopf}}^{n}(A, A)\right\}$ of vector spaces, or (iii) the graded vector space $\oplus_{n} \mathrm{CH}_{\mathrm{Hopf}}^{n}(A, A)$. It should be clear from the context what $\mathrm{CH}_{\mathrm{Hopf}}^{*}(A, A)$ means.

\section{Algebraic operad}

The purpose of this section is to show that the vector spaces $\mathrm{CH}_{\mathrm{Hopf}}^{*}(A, A)$ in the Hopf-Hochschild cochain complex of an $H$-module-algebra $A$ with self coefficients form an operad with multiplication (Theorem 3.1). 


\subsection{Operads}

Recall from $[\mathbf{1 6}, \mathbf{1 7}, \mathbf{1 8}]$ that a non- $\Sigma$ operad $\mathcal{O}=\{\mathcal{O}(n), \gamma, \mathrm{Id}\}$ consists of a sequence of vector spaces $\mathcal{O}(n)(n \geqslant 1)$ together with structure maps

$$
\gamma: \mathcal{O}(k) \otimes \mathcal{O}\left(n_{1}\right) \otimes \cdots \otimes \mathcal{O}\left(n_{k}\right) \rightarrow \mathcal{O}\left(n_{1}+\cdots+n_{k}\right),
$$

for $k, n_{1}, \ldots, n_{k} \geqslant 1$ and an identity element $\operatorname{Id} \in \mathcal{O}(1)$, satisfying the following two axioms.

1. The structure maps $\gamma$ are required to be associative, in the sense that

$$
\begin{aligned}
\gamma\left(\gamma\left(f ; g_{1, k}\right) ; h_{1, N}\right)= & \gamma\left(f ; \gamma\left(g_{1} ; h_{1, N_{1}}\right), \ldots,\right. \\
& \left.\gamma\left(g_{i} ; h_{N_{i-1}+1, N_{i}}\right), \ldots, \gamma\left(g_{k} ; h_{N_{k-1}+1, N_{k}}\right)\right) .
\end{aligned}
$$

Here $f \in \mathcal{O}(k), g_{i} \in \mathcal{O}\left(n_{i}\right), N=n_{1}+\cdots+n_{k}$, and $N_{i}=n_{1}+\cdots+n_{i}$. Given elements $x_{i}, x_{i+1}, \ldots$, the symbol $x_{i, j}$ is the abbreviation for the sequence $x_{i}$, $x_{i+1}, \ldots, x_{j}$ or $x_{i} \otimes \cdots \otimes x_{j}$ whenever $i \leqslant j$.

2. The maps

$$
\gamma(-; \mathrm{Id}, \ldots, \mathrm{Id}): \mathcal{O}(k) \rightarrow \mathcal{O}(k) \quad \text { and } \quad \gamma(\mathrm{Id} ;-): \mathcal{O}(k) \rightarrow \mathcal{O}(k)
$$

are both equal to the identity map on $\mathcal{O}(k)$ for each $k \geqslant 1$.

For the rest of this paper, we will refer to non- $\Sigma$ operads simply as operads.

\subsection{Operad morphism}

Let $\mathcal{O}$ and $\mathcal{P}$ be operads. An operad morphism $\phi: \mathcal{O} \rightarrow \mathcal{P}$ consists of a sequence of linear maps $\phi_{n}: \mathcal{O}(n) \rightarrow \mathcal{P}(n)$ such that

$$
\phi_{1}\left(\operatorname{Id}_{\mathcal{O}}\right)=\operatorname{Id}_{\mathcal{P}}
$$

and the diagram

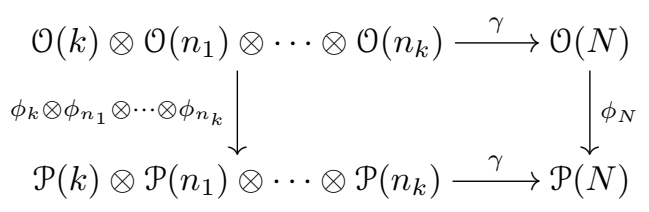

commutes for all $k, n_{1}, \ldots, n_{k} \geqslant 1$, where $N=n_{1}+\cdots+n_{k}$.

\subsection{Operad with multiplication}

Let $\mathcal{O}$ be an operad. A multiplication on $\mathcal{O}[\mathbf{1 0}$, Section 1.2] is an element $m \in \mathcal{O}(2)$ that satisfies

$$
\gamma(m ; m, \mathrm{Id})=\gamma(m ; \mathrm{Id}, m) .
$$

In this case, $(\mathcal{O}, m)$ is called an operad with multiplication. A multiplication on $\mathcal{O}$ is equivalent to an operad morphism $\mathcal{A} s \rightarrow \mathcal{O}$, where $\mathcal{A} s$ is the operad for associative algebras. The associative operad $\mathcal{A} s$ is generated by an element $\mu \in \mathcal{A} s(2)$, whose image under an operad morphism $\mathcal{A} s \rightarrow \mathcal{O}$ is a multiplication on $\mathcal{O}$.

\subsection{Operad with multiplication structure on $\mathrm{CH}_{\text {Hopf }}^{*}(A, A)$}

In what follows, in order to simplify the typography, we will sometimes write $\mathrm{C}(n)$ for the vector space $\mathrm{CH}_{\mathrm{Hopf}}^{n}(A, A)$. To show that the vector spaces $\mathrm{CH}_{\mathrm{Hopf}}^{*}(A, A)$ 
form an operad with multiplication, we first define the structure maps, the identity element, and the multiplication.

Structure maps For $k, n_{1}, \ldots, n_{k} \geqslant 1$, define a map

$$
\gamma: \mathcal{C}(k) \otimes \mathfrak{C}\left(n_{1}\right) \otimes \cdots \otimes \mathfrak{C}\left(n_{k}\right) \rightarrow \mathfrak{C}(N)
$$

by setting

$$
\begin{aligned}
& \gamma\left(f ; g_{1, k}\right)\left(a_{0, N+1}\right) \\
= & f\left(a_{0} \otimes g_{1}\left(1 \otimes a_{1, n_{1}} \otimes 1\right) \otimes \cdots \otimes g_{i}\left(1 \otimes a_{N_{i-1}+1, N_{i}} \otimes 1\right) \otimes \cdots \otimes a_{N+1}\right) .
\end{aligned}
$$

Here the notation is as in the definition of an operad above, and each $a_{i} \in A$.

Identity element Let $\mathrm{Id} \in \mathcal{C}(1)$ be the element such that

$$
\operatorname{Id}\left(a_{0} \otimes a_{1} \otimes a_{2}\right)=a_{0} a_{1} a_{2} .
$$

This is indeed an element of $\mathrm{C}(1)$, since the identity map on $A$ is $H$-linear.

Multiplication Let $\pi \in \mathcal{C}(2)$ be the element such that

$$
\pi\left(a_{0} \otimes a_{1} \otimes a_{2} \otimes a_{3}\right)=a_{0} a_{1} a_{2} a_{3} .
$$

This is indeed an element of $\mathrm{C}(2)$, since the multiplication map $A^{\otimes 2} \rightarrow A$ on $A$ is $H$-linear.

Theorem 3.1. The data

$$
\mathcal{C}=\{\mathrm{C}(n), \gamma, \mathrm{Id}\}
$$

forms an operad. Moreover, $\pi \in \mathcal{C}(2)$ is a multiplication on the operad $\mathcal{C}$.

Proof. It is immediate from (5) and (6) that $\gamma\left(-; \mathrm{Id}^{\otimes k}\right)$ and $\gamma(\mathrm{Id} ;-)$ are the identity map on $\mathcal{C}(k)$ for each $k \geqslant 1$.

To prove associativity of $\gamma$, we use the notations in the definition of an operad and compute as follows:

$$
\begin{aligned}
\gamma & \left(\gamma\left(f ; g_{1, k}\right) ; h_{1, N}\right)\left(a_{0} \otimes \cdots \otimes a_{M+1}\right) \\
\quad & =\gamma\left(f ; g_{1, k}\right)\left(a_{0} \otimes \cdots \otimes h_{j}\left(1 \otimes a_{M_{j-1}+1, M_{j}} \otimes 1\right) \otimes \cdots \otimes a_{M+1}\right) \\
& =f\left(a_{0} \otimes \cdots \otimes g_{i}\left(1 \otimes z_{i} \otimes 1\right) \otimes \cdots \otimes a_{M+1}\right) \\
& =\gamma\left(f ; \ldots, \gamma\left(g_{i} ; h_{N_{i-1}+1, N_{i}}\right), \ldots\right)\left(a_{0} \otimes \cdots \otimes a_{M+1}\right) .
\end{aligned}
$$

Here the element $z_{i}(1 \leqslant i \leqslant k)$ is given by

$$
\begin{aligned}
z_{i} & =\bigotimes_{l=N_{i-1}+1}^{N_{i}} h_{l}\left(1 \otimes a_{M_{l-1}+1, M_{l}} \otimes 1\right) \\
& =h_{N_{i-1}+1}\left(1 \otimes a_{M_{N_{i-1}}+1, M_{N_{i-1}+1}} \otimes 1\right) \otimes \cdots \otimes h_{N_{i}}\left(1 \otimes a_{M_{N_{i}-1}+1, M_{N_{i}}} \otimes 1\right) .
\end{aligned}
$$

This shows that $\gamma$ is associative and that $\mathcal{C}=\{\mathfrak{C}(n), \gamma, \operatorname{Id}\}$ is an operad.

To see that $\pi \in \mathcal{C}(2)$ is a multiplication on $\mathcal{C}$, one observes that both

$$
\gamma(\pi ; \pi, \mathrm{Id})\left(a_{0} \otimes \cdots \otimes a_{4}\right) \quad \text { and } \quad \gamma(\pi ; \mathrm{Id}, \pi)\left(a_{0} \otimes \cdots \otimes a_{4}\right)
$$

are equal to the product $a_{0} a_{1} a_{2} a_{3} a_{4}$.

This finishes the proof of Theorem 3.1. 


\subsection{Relationship with the Hochschild complex}

Here we observe that the operad with multiplication $(\mathcal{C}, \pi)$ induces the usual operad with multiplication structure on the Hochschild complex.

Recall that the Hochschild complex

$$
C^{*}(A, A)=\left\{C^{n}(A, A)=\operatorname{Hom}_{K}\left(A^{\otimes n}, A\right)\right\}
$$

is an operad with multiplication $\mu_{A}$ [10]. Indeed, $C^{*}(A, A)$ is the endomorphism operad of $A$. Its structure maps $\gamma$ are given by

$$
\gamma\left(f ; g_{1, k}\right)\left(a_{1, N}\right)=f\left(\cdots \otimes g_{i}\left(a_{N_{i-1}+1, N_{i}}\right) \otimes \cdots\right)
$$

for $f \in C^{n}(A, A), g_{i} \in C^{n_{i}}(A, A)$, and $a_{j} \in A$. The notations are as in section 3.1 with $N_{0}=0$. The identity element in $C^{1}(A, A)$ is the identity map on $A$.

Denote by

$$
\phi_{\text {Hopf }}: \mathcal{A} s \rightarrow \mathrm{CH}_{\text {Hopf }}^{*}(A, A) \quad \text { and } \quad \phi: \mathcal{A} s \rightarrow C^{*}(A, A)
$$

the operad morphisms corresponding to the operads with multiplication $\mathrm{CH}_{\mathrm{Hopf}}^{*}(A, A)$ and $C^{*}(A, A)$, respectively. The following observation says that $\phi$ is induced by $\phi_{\mathrm{Hopf}}$.

Corollary 3.2. The operad with multiplication $C^{*}(A, A)$ is induced by $\mathrm{CH}_{\mathrm{Hopf}}^{*}(A, A)$ in the sense that there is an operad morphism $\rho: \mathrm{CH}_{\mathrm{Hopf}}^{*}(A, A) \rightarrow C^{*}(A, A)$ such that the diagram

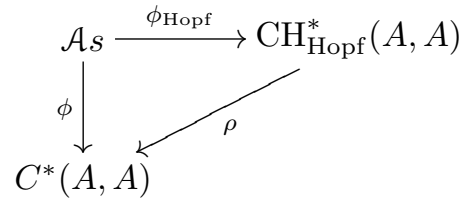

commutes.

Proof. Let us abbreviate $C^{n}(A, A)$ to $C^{n}$ and $\mathrm{CH}_{\text {Hopf }}^{n}(A, A)$ to $\mathcal{C}(n)$. The component map $\rho: \mathcal{C}(n) \rightarrow C^{n}$ is defined as

$$
\rho(g)\left(a_{1, n}\right)=g\left(1 \otimes a_{1, n} \otimes 1\right)
$$

for $a_{i} \in A$. To see that the map $\rho$ is an operad morphism, first observe that it preserves the identity elements because

$$
\rho(\operatorname{Id})(a)=\operatorname{Id}(1 \otimes a \otimes 1)=a
$$

by (6). Moreover, the commutativity of the diagram (3) for $\rho$ follows from the fact that both $\rho\left(\gamma\left(f ; g_{1, k}\right)\right)\left(a_{1, N}\right)$ and $\gamma\left(\rho(f) ; \rho\left(g_{1, k}\right)\right)\left(a_{1, N}\right)$ are equal to

$$
f\left(1 \otimes \cdots \otimes g_{i}\left(1 \otimes a_{N_{i-1}+1, N_{i}} \otimes 1\right) \otimes \cdots \otimes 1\right) .
$$

Here $f \in \mathcal{C}(k), g_{i} \in \mathcal{C}\left(n_{i}\right), N=n_{1}+\cdots+n_{k}, N_{0}=0$, and $a_{j} \in A$.

Now we show that the diagram (8) commutes. Note that the operad morphism $\phi$ is uniquely determined by the property $\phi(\mu)=\mu_{A}$, where $\mu \in \mathcal{A} s(2)$ is the generator for the associative operad. Likewise, we have $\phi_{\text {Hopf }}(\mu)=\pi$. Since we have

$$
\rho(\pi)\left(a_{1} \otimes a_{2}\right)=\pi\left(1 \otimes a_{1} \otimes a_{2} \otimes 1\right)=a_{1} a_{2}
$$

by (7), we conclude that $\rho(\pi)=\mu_{A}$ and $\rho\left(\phi_{\text {Hopf }}(\mu)\right)=\mu_{A}$. This implies by uniqueness that $\phi=\rho \circ \phi_{\text {Hopf }}$. 
DONALD YAU

\section{Brace algebra}

The purpose of this section is to show that the graded vector space $\mathrm{CH}_{\mathrm{Hopf}}^{*}(A, A)$ admits the structure of a brace algebra with multiplication. Explicit formulas of the brace operations are given in (11).

\subsection{Brace algebra}

For a graded vector space $V=\oplus_{n=1}^{\infty} V^{n}$ and an element $x \in V^{n}$, set $\operatorname{deg} x=n$ and $|x|=n-1$. Elements in $V^{n}$ are said to have degree $n$.

Recall from [10, Definition 1] that a brace algebra is a graded vector space $V=$ $\oplus V^{n}$ together with a collection of brace operations $x\left\{x_{1}, \ldots, x_{n}\right\}$ of degree $-n$, satisfying the associativity axiom:

$$
\begin{array}{r}
x\left\{x_{1, m}\right\}\left\{y_{1, n}\right\}=\sum_{0 \leqslant i_{1} \leqslant \cdots \leqslant i_{m} \leqslant n}(-1)^{\varepsilon} x\left\{y_{1, i_{1}}, x_{1}\left\{y_{i_{1}+1, j_{1}}\right\}, y_{j_{1}+1}, \ldots, y_{i_{m}},\right. \\
\left.x_{m}\left\{y_{i_{m}+1, j_{m}}\right\}, y_{j_{m}+1, n}\right\} .
\end{array}
$$

Here the sign is given by $\varepsilon=\sum_{p=1}^{m}\left(\left|x_{p}\right| \sum_{q=1}^{i_{p}}\left|y_{q}\right|\right)$.

\subsection{Brace algebra with multiplication}

Let $V=\oplus V^{n}$ be a brace algebra. A multiplication on $V[\mathbf{1 0}$, Section 1.2] is an element $m \in V^{2}$ such that

$$
m\{m\}=0 .
$$

In this case, we call $V=(V, m)$ a brace algebra with multiplication.

\subsection{Brace algebra from operad}

Suppose that $\mathcal{O}=\{\mathcal{O}(n), \gamma, \operatorname{Id}\}$ is an operad. Define the following operations on the graded vector space $\mathcal{O}=\oplus \mathcal{O}(n)$ :

$$
x\left\{x_{1}, \ldots, x_{n}\right\} \stackrel{\text { def }}{=} \sum(-1)^{\varepsilon} \gamma\left(x ; \mathrm{Id}, \ldots, \mathrm{Id}, x_{1}, \mathrm{Id}, \ldots, \mathrm{Id}, x_{n}, \mathrm{Id}, \ldots, \mathrm{Id}\right) .
$$

Here the sum runs over all possible substitutions of $x_{1}, \ldots, x_{n}$ into $\gamma(x ; \ldots)$ in the given order. The sign is determined by $\varepsilon=\sum_{p=1}^{n}\left|x_{p}\right| i_{p}$, where $i_{p}$ is the total number of inputs in front of $x_{p}$. Note that

$$
\operatorname{deg} x\left\{x_{1}, \ldots, x_{n}\right\}=\operatorname{deg} x-n+\sum_{p=1}^{n} \operatorname{deg} x_{p},
$$

so the operation (10) is of degree $-n$.

Proposition 1 in [10] establishes that the operations (10) make the graded vector space $\oplus \mathcal{O}(n)$ into a brace algebra. Moreover, a multiplication on the operad $\mathcal{O}$ in the sense of $\S 3.3$ is equivalent to a multiplication on the brace algebra $\oplus \mathcal{O}(n)$. In fact, for an element $m \in \mathcal{O}(2)$, one has that

$$
m\{m\}=\gamma(m ; m, \mathrm{Id})-\gamma(m ; \mathrm{Id}, m) .
$$

It follows that the condition (4) is equivalent to (9). In other words, an operad with multiplication $(\mathcal{O}, m)$ gives rise to a brace algebra with multiplication $(\oplus \mathcal{O}(n), m)$. Combining this discussion with Theorem 3.1, we obtain the following result. 
Corollary 4.1. The graded vector space $\mathrm{CH}_{\mathrm{Hopf}}^{*}(A, A)$ is a brace algebra with brace operations as in (10) and multiplication $\pi(7)$.

The brace operations on $\mathrm{CH}_{\mathrm{Hopf}}^{*}(A, A)$ can be described more explicitly as follows. For $f \in \mathcal{C}(k)$ and $g_{i} \in \mathcal{C}\left(m_{i}\right)(1 \leqslant i \leqslant n)$, we have

$$
f\left\{g_{1}, \ldots, g_{n}\right\}=\sum(-1)^{\varepsilon} \gamma\left(f ; \operatorname{Id}^{r_{1}}, g_{1}, \operatorname{Id}^{r_{2}}, g_{2}, \ldots, \mathrm{Id}^{r_{n}}, g_{n}, \mathrm{Id}^{r_{n+1}}\right),
$$

where $\mathrm{Id}^{r}=\mathrm{Id}^{\otimes r}$. Here the $r_{j}$ are given by

$$
r_{j}= \begin{cases}i_{1} & \text { if } j=1, \\ i_{j}-i_{j-1}-1 & \text { if } 2 \leqslant j \leqslant n, \\ k-i_{n}-1 & \text { if } j=n+1,\end{cases}
$$

and

$$
\varepsilon=\sum_{p=1}^{n}\left(m_{p}-1\right) i_{p}
$$

Write $M=\sum_{i=1}^{n} m_{i}$ and $M_{j}=\sum_{i=1}^{j} m_{i}$. For an element $a_{0, k+M-n+1}$ in $A^{\otimes(k+M-n)}$, we have

$$
\begin{aligned}
f\left\{g_{1, n}\right\}\left(a_{0, k+M-n+1}\right)=\sum & (-1)^{\varepsilon} f\left(a_{0, i_{1}} \otimes g_{1}\left(1 \otimes a_{i_{1}+1, i_{1}+m_{1}} \otimes 1\right) \otimes \cdots\right. \\
& \otimes a_{i_{j-1}+M_{j-1}-(j-1)+2, i_{j}+M_{j-1}-j+1} \\
& \otimes g_{j}\left(1 \otimes a_{i_{j}+M_{j-1}-j+2, i_{j}+M_{j}-j+1} \otimes 1\right) \otimes \cdots \\
& \left.\otimes a_{i_{n}+M-n+2, k+M-n+1}\right) .
\end{aligned}
$$

\section{Homotopy Gerstenhaber algebra}

The purpose of this section is to observe that the brace algebra with multiplication structure on $\mathrm{CH}_{\mathrm{Hopf}}^{*}(A, A)$ induces a homotopy Gerstenhaber algebra structure (Corollary 5.1). The underlying cochain complex of this homotopy Gerstenhaber algebra is canonically isomorphic to the Hopf-Hochschild cochain complex of $A$ (Corollary 5.3).

\subsection{Homotopy $G$-algebra}

Recall from [10, Definition 2] that a homotopy $G$-algebra $(V, d, \cup)$ consists of a brace algebra $V=\oplus V^{n}$, a degree +1 differential $d$, and a degree 0 associative $\cup$ product that makes $V$ into a differential graded algebra, satisfying the following two conditions.

1. The $\cup$-product is required to satisfy the condition

$$
\left(x_{1} \cup x_{2}\right)\left\{y_{1, n}\right\}=\sum_{k=0}^{n}(-1)^{\varepsilon} x_{1}\left\{y_{1, k}\right\} \cup x_{2}\left\{y_{k+1, n}\right\},
$$

where $\varepsilon=\left|x_{2}\right| \sum_{p=1}^{k}\left|y_{p}\right|$, for $x_{i}, y_{j} \in V$. 
2. The differential is required to satisfy the condition

$$
\begin{aligned}
d( & \left.x\left\{x_{1, n+1}\right\}\right)-(d x)\left\{x_{1, n+1}\right\} \\
& -(-1)^{|x|} \sum_{i=1}^{n+1}(-1)^{\left|x_{1}\right|+\cdots+\left|x_{i-1}\right|} x\left\{x_{1}, \ldots, d x_{i}, \ldots, x_{n+1}\right\} \\
= & (-1)^{|x|\left|x_{1}\right|+1} x_{1} \cup x\left\{x_{2, n+1}\right\}-x\left\{x_{1, n}\right\} \cup x_{n+1} \\
& +(-1)^{|x|} \sum_{i=1}^{n}(-1)^{\left|x_{1}\right|+\cdots+\left|x_{i-1}\right|} x\left\{x_{1}, \ldots, x_{i} \cup x_{i+1}, \ldots, x_{n+1}\right\} .
\end{aligned}
$$

\subsection{Homotopy $G$-algebra from brace algebra with multiplication}

A brace algebra with multiplication $V=(V, m)$ gives rise to a homotopy $G$-algebra $(V, d, \cup)[\mathbf{1 0}$, Theorem 3], where the $\cup$-product and the differential are defined as:

$$
\begin{aligned}
x \cup y & =(-1)^{\operatorname{deg} x} m\{x, y\}, \\
d(x) & =m\{x\}-(-1)^{|x|} x\{m\} .
\end{aligned}
$$

In particular, this applies to the brace algebra $\mathrm{CH}_{\mathrm{Hopf}}^{*}(A, A)$ with multiplication $\pi$ (Corollary 4.1).

Corollary 5.1. For an $H$-module-algebra $A, \mathrm{C}=\left(\mathrm{CH}_{\mathrm{Hopf}}^{*}(A, A), d, \cup\right)$ is a homotopy G-algebra.

\subsection{Comparing differentials}

There are two differentials on the graded vector space $\mathrm{CH}_{\mathrm{Hopf}}^{*}(A, A)$, namely, the differential $d^{n}$ (12) induced by the multiplication $\pi$ and the Hopf-Hochschild differential $d_{\mathrm{CH}}^{n}(2)$. The following result ensures that the cohomology modules defined by these two differentials are the same.

Theorem 5.2. The equality

$$
d_{\mathrm{CH}}^{n}=(-1)^{n+1} d^{n}
$$

holds for each $n$.

Proof. Pick $f \in \mathrm{CH}_{\mathrm{Hopf}}^{n}(A, A)$. Then we have

$$
\begin{aligned}
d^{n} f & =\pi\{f\}+(-1)^{n} f\{\pi\} \\
& =(-1)^{n-1} \gamma(\pi ; \mathrm{Id}, f)+\gamma(\pi ; f, \mathrm{Id})+(-1)^{n} \sum_{i=1}^{n}(-1)^{i-1} \gamma\left(f ; \mathrm{Id}^{i-1}, \pi, \operatorname{Id}^{n-i}\right) .
\end{aligned}
$$

It follows that

$$
(-1)^{n+1} d^{n} f=\gamma(\pi ; \mathrm{Id}, f)+(-1)^{n+1} \gamma(\pi ; f, \mathrm{Id})+\sum_{i=1}^{n}(-1)^{i} \gamma\left(f ; \mathrm{Id}^{i-1}, \pi, \mathrm{Id}^{n-i}\right) .
$$

Observe that [14]

$$
g\left(a_{0, n+1}\right)=a_{0} g\left(1 \otimes a_{1, n} \otimes 1\right) a_{n+1}
$$

for $g \in \mathrm{CH}_{\mathrm{Hopf}}^{n}(A, A)$. Using (14) and applying the various terms in (13) to an element 


$$
\begin{aligned}
a_{0, n+2} \in \mathrm{CB}_{n+1}(A)=A^{\otimes(n+3)}, \text { we obtain } & \\
\gamma(\pi ; \mathrm{Id}, f)\left(a_{0, n+2}\right) & =f\left(a_{0} a_{1} \otimes a_{2, n+2}\right), \\
\gamma(\pi ; f, \mathrm{Id})\left(a_{0, n+2}\right) & =f\left(a_{0, n} \otimes a_{n+1} a_{n+2}\right), \\
\gamma\left(f ; \mathrm{Id}^{i-1}, \pi, \mathrm{Id}^{n-i}\right)\left(a_{0, n+2}\right) & =f\left(a_{0, i-1} \otimes a_{i} a_{i+1} \otimes a_{i+2, n+2}\right) .
\end{aligned}
$$

The Theorem now follows immediately from (13) and (15).

Corollary 5.3. There is an isomorphism of cochain complexes,

$$
\begin{aligned}
\left(\mathrm{CH}_{\mathrm{Hopf}}^{*}(A, A), d_{\mathrm{CH}}\right) & \cong\left(\mathrm{CH}_{\mathrm{Hopf}}^{*}(A, A), d\right) \\
x & \mapsto(-1)^{\frac{n(n+1)}{2}} x,
\end{aligned}
$$

for $x \in \mathrm{CH}_{\mathrm{Hopf}}^{n}(A, A)$. In particular, the cohomology modules on $\mathrm{CH}_{\mathrm{Hopf}}^{*}(A, A)$ defined by the differentials $d_{\mathrm{CH}}$ and $d$ are equal.

\section{Gerstenhaber algebra}

The purpose of this section is to observe that the homotopy $G$-algebra structure on $\mathrm{CH}_{\mathrm{Hopf}}^{*}(A, A)$ gives rise to a $G$-algebra structure on the Hopf-Hochschild cohomology modules $H H_{\mathrm{Hopf}}^{*}(A, A)$. Explicit formulas of these $G$-algebra operations are given in (16).

\subsection{Gerstenhaber algebra}

Recall from [10, Section 2.2] that a $G$-algebra $(V, \cup,[-,-])$ consists of a graded vector space $V=\oplus V^{n}$, a degree 0 associative $U$-product, and a degree -1 graded Lie bracket

$$
[-,-]: V^{m} \otimes V^{n} \rightarrow V^{m+n-1},
$$

satisfying the following two conditions:

$$
\begin{aligned}
x \cup y & =(-1)^{\operatorname{deg} x \operatorname{deg} y} y \cup x, \\
{[x, y \cup z] } & =[x, y] \cup z+(-1)^{|x| \operatorname{deg} y} y \cup[x, z] .
\end{aligned}
$$

In other words, the $\cup$-product is graded commutative, and the Lie bracket is a graded derivation for the $\cup$-product. In particular, a $G$-algebra is a graded version of a Poisson algebra. This algebraic structure was first studied by Gerstenhaber [8].

\section{2. $\quad G$-algebra from homotopy $G$-algebra}

If $(V, d, \cup)$ is a homotopy $G$-algebra, one can define a degree -1 operation on $V$ as

$$
[x, y] \stackrel{\text { def }}{=} x\{y\}-(-1)^{|x||y|} y\{x\} .
$$

Passing to cohomology, $\left(H^{*}(V, d), \cup,[-,-]\right)$ becomes a $G$-algebra ([10] Corollary 5 and its proof).

Combining the previous paragraph with Corollary 5.1 and Corollary 5.3, we obtain the following result.

Corollary 6.1. The Hopf-Hochschild cohomology modules $H H_{\mathrm{Hopf}}^{*}(A, A)$ of an $H$ module-algebra $A$ admits the structure of a G-algebra. 
This $G$-algebra can be described on the cochain level more explicitly as follows. Pick $\varphi \in \mathrm{CH}_{\text {Hopf }}^{n}(A, A)$ and $\psi \in \mathrm{CH}_{\text {Hopf }}^{m}(A, A)$. Then

$$
\begin{aligned}
(\psi \cup \varphi)\left(a_{0, m+n+1}\right) & =(-1)^{m+n-1} \psi\left(a_{0, m} \otimes 1\right) \varphi\left(1 \otimes a_{m+1, m+n+1}\right), \\
{[\psi, \varphi] } & =\psi\{\varphi\}-(-1)^{(m-1)(n-1)} \varphi\{\psi\} .
\end{aligned}
$$

Writing $a=a_{0, m+n}$, we have

$$
\begin{aligned}
& \psi\{\varphi\}(a)=\sum_{i=1}^{m}(-1)^{(i-1)(n-1)} \psi\left(a_{0, i-1} \otimes \varphi\left(1 \otimes a_{i, i+n-1} \otimes 1\right) \otimes a_{i+n, m+n}\right), \\
& \varphi\{\psi\}(a)=\sum_{j=1}^{n}(-1)^{(j-1)(m-1)} \varphi\left(a_{0, j-1} \otimes \psi\left(1 \otimes a_{j, j+m-1} \otimes 1\right) \otimes a_{j+m, m+n}\right) .
\end{aligned}
$$

In particular, if $m=n=1$, then the bracket operation

$$
[\psi, \varphi]\left(a_{0,2}\right)=\psi\left(a_{0} \otimes \varphi\left(1 \otimes a_{1} \otimes 1\right) \otimes a_{2}\right)-\varphi\left(a_{0} \otimes \psi\left(1 \otimes a_{1} \otimes 1\right) \otimes a_{2}\right)
$$

gives $H H_{\text {Hopf }}^{1}(A, A)$ a Lie algebra structure. There is another description of this Lie algebra in terms of (inner) derivations in [14, Proposition 3.9].

\section{Deligne's Conjecture for module-algebras}

The purpose of this section is to observe that a version of Deligne's Conjecture holds for the Hopf-Hochschild cochain complex of a module-algebra. The original Deligne's Conjecture for Hochschild cohomology is as follows.

Deligne's Conjecture ([6]). The Hochschild cochain complex $C^{*}(R, R)$ of an associative algebra $R$ is an algebra over a suitable chain model of May's little squares operad $\mathrm{C}_{2}[\mathbf{1 7}]$.

A positive answer to Deligne's conjecture was given by, among others, McClure and Smith [19, Theorem 1.1] and Kaufmann [13, Theorem 4.2.2]. There is an operad $\mathcal{H}$ whose algebras are the brace algebras with multiplication (section 4.2). For an associative algebra $R$, the Hochschild cochain complex $C^{*}(R, R)$ is a brace algebra with multiplication and hence an $\mathcal{H}$-algebra. McClure and Smith showed that $\mathcal{H}$ is quasi-isomorphic to the chain operad $\mathcal{S}$ obtained from the little squares operad $\mathcal{C}_{2}$ by applying the singular chain functor, thereby proving Deligne's Conjecture.

It has been observed that the Hopf-Hochschild cochain complex $\mathrm{CH}_{\mathrm{Hopf}}^{*}(A, A)$ is a brace algebra with multiplication (Corollary 4.1). Therefore, we can use the result of McClure and Smith [19, Theorem 1.1] to obtain the following version of Deligne's Conjecture for module-algebras.

Corollary 7.1 (Deligne's Conjecture for module-algebras). The Hopf-Hochschild complex $\mathrm{CH}_{\mathrm{Hopf}}^{*}(A, A)$ of an $H$-module-algebra $A$ is an algebra over the McClureSmith operad $\mathcal{H}$ that is a chain model for the little squares operad $\mathcal{C}_{2}$. 
OPERATIONS ON THE HOPF-HOCHSCHILD COMPLEX FOR MODULE-ALGEBRAS

271

\section{References}

[1] R. Akbarpour and M. Khalkhali, Hopf algebra equivariant cyclic homology and cyclic homology of crossed product algebras, J. reine angew. Math. 559 (2003), $137-152$.

[2] R. Akbarpour and M. Khalkhali, Cyclic cohomology of crossed coproduct coalgebras, preprint, arXiv:math.KT/0107166.

[3] R. Akbarpour and M. Khalkhali, Cyclic homology of Hopf comodule algebras and Hopf module coalgebras, Comm. Alg. 31 (2003), 5653-5671.

[4] J.M. Boardman, Stable operations in generalized cohomology, in: Handbook of algebraic topology, 585-686, North-Holland, Amsterdam, 1995.

[5] S. Dăsălescu, C. Năstăsescu and Ş. Raianu, Hopf algebra: An introduction, Marcel Dekker, New York, 2001.

[6] P. Deligne, Letter to Stasheff, Gerstenhaber, May, Schechtman, Drinfeld, May 17, 1993.

[7] D.B.A. Epstein and N.E. Steenrod, Cohomology operations, Princeton Univ. Press, Princeton, NJ, 1962.

[8] M. Gerstenhaber, The cohomology structure of an associative ring, Ann. Math. 78 (1963), 267-288.

[9] M. Gerstenhaber, On the deformation of rings and algebras, Ann. Math. 79 (1964), 59-103.

[10] M. Gerstenhaber and A.A. Voronov, Homotopy $G$-algebras and moduli space operad, Int. Math. Res. Notices 1995, 141-153.

[11] G. Hochschild, On the cohomology groups of an associative algebra, Ann. Math. 46 (1945), 58-67.

[12] C. Kassel, Quantum groups, Springer-Verlag, New York, 1995.

[13] R.M. Kaufmann, On spineless cacti, Deligne's conjecture and Connes-Kreimer's Hopf algebra, Topology 46 (2007), 39-88.

[14] A. Kaygun, Hopf-Hochschild (co)homology of module algebras, Homology Homotopy Appl. 9 (2007), 451-472.

[15] P.S. Landweber, Cobordism operations and Hopf algebras, Trans. Amer. Math. Soc. 129 (1967), 94-110.

[16] M. Markl, S. Shnider and J. Stasheff, Operads in Algebra, Topology and Physics, Amer. Math. Soc., Providence, RI, 2002.

[17] J.P. May, The geometry of iterated loop spaces, Lectures Notes in Math. 271, Springer-Verlag, Berlin-New York, 1972.

[18] J.P. May, Definitions: operads, algebras and modules, Contemp. Math. 202 (1997), 1-7.

[19] J.E. McClure and J.H. Smith, A solution of Deligne's Hochschild cohomology conjecture, Contemp. Math. 293 (2002), 153-193.

[20] J. Milnor, The Steenrod algebra and its dual, Ann. Math. 67 (1958), 150-171.

[21] S. Montgomery, Hopf algebras and their actions on rings, Amer. Math. Soc., Providence, RI, 1993. 
[22] S.P. Novikov, Methods of algebraic topology from the point of view of cobordism theory, Izv. Akad. Nauk SSSR Ser. Mat. 31 (1967), 855-951.

[23] M. Sweedler, Hopf algebras, W.A. Benjamin, New York, 1969.

[24] D. Yau, Deformation bicomplex of module-algebras, Homology Homotopy Appl. 10 (2008), 97-128.

Donald Yau dyau@math.ohio-state.edu

Department of Mathematics, The Ohio State University Newark, 1179 University Drive, Newark, OH 43055, USA 